in genetically affected subjects without echocardiographic evidence of left ventricular hypertrophy. Em Heart J. 1998; 19: 489-498.

9. Seidman JG, Seidman C. The genetic basis for cardiomyopathy: from mutation identification to mechanistic paradigms. Cell. 2001; 104: 557-567.

10. Yakushin SS, Filippov EV. Gipertroficheskaya kardiomiopatiya: rezul'taty pyatiletnego nablyudeniya [Hypertrophic cardiomyopathy: five-year follow-up]. Bolezni serdca i sosudov [Heart and vascular disease]. 2006; 2: 34-38.

11. SV Moiseev, Moiseev VS, Kabalova ZhD. Bolezni serdca: Rukovodstvo dlya vrachej [Heart diseases: A guide for doctors]. Moskva: MIA [Moscow: Medical News Agency]. 2008; $528 \mathrm{p}$.

12. Jeanrenaud X, Kappenberger L, Maron BJ, et al. Dual-chamber pacing for hypertrophic obstructive cardiomyopathy. Diagnosis and management of hypertrophic cardomyopathy. Blackwell: Futura. 2004; 246-258.

13. Sorajja P, Ommen SR, Maron BJ, et al. United States perspectives on the role of dual-chamber pacing in patients with hypertrophic cardiomyopathy. Diagnosis and management of hypertrophic cardomyopathy. Blackwell: Futura. 2004; 236-245.

14. Amosova EN. Kardiomiopatii [Cardiomyopathies]. Kiev: Kniga Plyus [Kiev: Book Plus]. 1999; 421 p.

15. Wan CK, Dearani JA, Sundt TM, et al. What is the best surgical treatment for obstructive hypertrophic cardiomyopathy and degenerative mitral valve regurgitation? Ann Thorac Surg. 2009; 88: 727-732.

16. Gerchikova TN, Topolyanskij MK. Bolezni serdca [Heart Diseases]. Moskva: Enciklopediya [Moscow: Encyclopedia]. 2006; 544 p.

17. Yetman AT, McCrindle BW, MacDonald C, et al. Myocardial bridging in children with hypertrophic cardiomyopathy - a risk factor for sudden death. N Engl J Med. 2004; 339: 1201-1209.

18. Belenkov YuN, Privalova EV, Kaplunova VYu. Gipertroficheskaya kardiomiopatiya: Rukovodstvo dlya vrachej [Hypertrophic cardiomyopathy: a guide for doctors]. Moskva: GEOTAR-Media [Moscow: GEOTAR-Media]. 2011; 392 p.

19. Spirito P, Seidman CE, McKenna WJ, Maron BJ. The management of hypertrophic cardiomyopathy. $\mathrm{N}$ Engl J Med. 1997; 336: 775-785.

20. Sherrid MV, Chaudhry FA, Swistel DG. Obstructive hypertrophic cardiomyopathy: echocardiography, pathophysiology, and the continuing evolution of surgery for obstruction. Ann Thorac Surg. 2003; 75 (2): 620-632.

21. Maron BJ, Spirito P. Implications of left ventricular remodeling in hypertrophic cardiomyopathy. Am J Cardiol. 1998; 81: 1339-1344.

22. Maron BJ, Casey SA, Poliac LC, et al. Clinical course of hypertrophic cardiomyopathy in a regional United States cohort. JAMA. 1999; 281: 650-655.

23. Jeanrenaud X, Kappenberger L, Maron BJ. Dual-chamber pacing for hypertrophic obstructive cardiomyopathy; Diagnosis and management of hypertrophic cardomyopathy. Blackwell: Futura. 2004; 246-258.
24. Cheitlin MD, Armstrong WF, Aurigemma GP, et al. ACC/AHA/ASE 2003 guideline update for the clinical application of echocardiography: summary article: a report of the American College of Cardiology/American Heart Association Task Force on Practice Guidelines (ACC/ AHA/ASE Committee to Update the 1997 Guidelines for the Clinical Application of Echocardiography). Circulation. 2003; 108: 1146-1162.

25. Bokeriya LA, Borisov KV. Obstruktivnaya gipertroficheskaya kardiomiopatiya: metody hirurgicheskoj korrekcii [Obstructive hypertrophic cardiomyopathy: surgical correction methods]. Grudnaya i serdechno-sosudistaya hirurgiya [Thoracic and cardiovascular surgery]. 1997; 1: $61-65$.

26. Williams WG. Combined anterior mitral leaflet extension and myectomy in hypertrophic obsctructive cardiomyopathy. Letter to the Editor, Circulation. 2004; 109: 324.

27. Matsuda $\mathrm{H}$. Transatrial and transmitral myectomy for hypertrophic obstructive cardiomyopathy of the left ventricle. Oper Techn Thor Cardiovasc Surg. 2004; 9 (4): 304-309.

28. Stassano P, Tommaso L, Triggiani D, et al. Mitral valve replacement and limited myectomy for hypertrophic obstructive cardiomyopathy. Tex Heart Inst. 2004; 31 (2): 137-142.

29. Dearani JA, Danielson GK. Septal myectomy for obstructive hypertrophic cardiomyopathy. Operat Techn Thor Cardiovasc Surg. 2004; 9 (4): 278-292.

30. Shulte HD, Bircks WH. Surgery for hypertrophic obstructive cardiomyopathy (HOCM): the extended transaortic subvalvular myectomy (TSM) approach. Operat Techn Thor Cardiovasc Surg. 2004; 9 (4): 293-303.

31. Bokeriya LA, Borisov KV, Sinev AF. Original'nyj sposob hirurgicheskogo lecheniya gipertroficheskoj obstruktivnoj kardiomiopatii [An original method of surgical treatment of hypertrophic obstructive cardiomyopathy]. Grudnaya i serdechno-sosudistaya hirurgiya [Thoracic and cardiovascular surgery]. 1998; 2: 4-10.

32. Bokeriya LA, Borisov KV, Sinev AF. Uluchshenie diastolicheskoj funkcii levogo i pravogo zheludochkov serdca posle hirurgicheskoj korrekcii gipertroficheskoj obstruktivnoj kardiomiopatii pri pomoshchi original'nogo sposoba [Improving the diastolic function of the left and right ventricles of the heart after surgical correction of hypertrophic obstructive cardiomyopathy using the original method]. Grudnaya i serdechno-sosudistaya hirurgiya [Thoracic and cardiovascular surgery]. 1999; 4: 4-10.

33. Faber L, Meissner A, Ziemssen P, Seggewiss $H$. Percutaneous transluminal septal myocardial ablation for hypertrophic obstructive cardiomyopathy: long term follow up of the first series of 25 patients. Heart. 2000; 83: 326-331.

34. Heldman AW, Heldman AW, Wu KC, Abraham TP, Cameron DE. Myectomy or alcohol septal ablation surgery and percutaneous intervention go another round. J Am Coll Cardiol. 2007; 49 (3): 358-360.

МИНУЛЛИНА НИНА КОНСтАНтИНОВНА, канд. мед. наук, доцент кафедры_акушерства и гинекологии им. проф. B.С. Груздева, ФГБОУ ВО «Казанский государственный медицинский университет» Минздрава России, Россия, 420012, Казань, ул. Бутлерова, 49, e-mail: nina-constan@mail.ru 
АХМЕТОВА ДИНАРА ИЛДУСОВНА, студентка VI курса лечебного факультета ФГБОУ ВО «Казанский государственный медицинский университет» Минздрава России, Россия, 420012, Казань, ул. Бутлерова, 49,

e-mail: dinara.akhmetova.2496@mail.ru

Реферат. Цель исследования - изучить проблему варикозной болезни вен таза в структуре синдрома хронических тазовых болей, современные принципы диагностики и лечения данной патологии. Материал и методы. Были изучены российские и зарубежные научные публикации по данной тематике за последние 10 лет. Результаты и их обсуждение. В данной статье представлена современная концепция основных этиопатогенетических механизмов, диагностических принципов и лечения варикозной болезни вен таза как одной из причин хронической тазовой боли. Этиологические факторы могут быть разнообразны. Это как гинекологическая патология, так и поражение сосудов вследствие системных проявлений, нарушения гормонального фона, воспалительных процессов, генетической предрасположенности, анатомо-фризиологических особенностей и других причин. Патогенетически варикозная трансформация протекает по двум основным принципам: клапанная недостаточность и формирование обструктивных нарушений. Это приводит, в свою очередь, к патологическому рефлюксу и застойным явлениям в венозной системе таза. Основной причиной обращения женщин к специалистам является хроническая тазовая боль, которая характеризуется как нециклическая боль продолжительностью более 6 мес, локализующаяся в малом тазу, снижающая качество жизни пациентки. Основная сложность заключается именно в диффференциальной диагностике. Под маской синдрома тазовой боли может скрываться огромное количество как гинекологических, так и не гинекологических заболеваний. Своевременное предположение и проведение адекватной диагностической маршрутизации пациентки позволят быстрее установить верный диагноз и снизить риск диагностических и, как следствие, лечебных ошибок. Выводы. Несмотря на то что варикозная болезнь вен таза является известной патологией и изучается уже много лет, до сих пор не существует четкого представления о природе этого заболевания. Данная патология имеет мультидисциплинарный характер и требует слаженной и преемственной работы специалистов. Ввиду отсутствия специфики в клинической картине и надежных маркеров заболевания многие женщины проходят длительный и тяжелый путь от первичного обращения до постановки окончательного диагноза.

Ключевые слова: варикозная болезнь вен таза, синдром хронических тазовых болей.

Для ссылки: Минуллина, Н.К. Современные представления о варикозной болезни вен таза в структуре синдрома хронических тазовых болей: диагностика и лечение (обзор) / Н.К. Минуллина, Д.И. Ахметова // Вестник современной клинической медицины. - 2020. - Т. 13, вып. 2. - С.62-69. DOI: 10.20969/VSKM.2020.13(2).62-69.

\title{
MODERN UNDERSTANDING OF PELVIC VARICOSE VEIN DISEASE IN THE STRUCTURE OF CHRONIC PELVIC PAIN SYNDROME: DIAGNOSIS AND TREATMENT (review)
}

MINULLINA NINA K., C. Med. Sci., associate professor of the Department of obstetrics and gynecology of Kazan State Medical University, Russia, 420012, Kazan, Butlerov str., 49, e-mail: nina-constan@mail.ru AKHMETOVA DINARA I., $6^{\text {th }}$ year student of Faculty of general medicine of Kazan State Medical University, Russia, 420012 Kazan, Butlerov str., 49, e-mail: dinara.akhmetova.2496@mail.ru

\begin{abstract}
Aim. The aim of the study was to investigate the problem of pelvic varicose vein disease in the structure of chronic pelvic pain syndrome, as well as the modern principles of diagnosis and treatment of this disease. Material and methods. Russian and foreign scientific publications on this topic for the last 10 years have been studied. Results and discussion. This article presents a modern concept of basic etiopathogenetic mechanisms, diagnostic principles and treatment of pelvic varicose vein disease as one of the causes of chronic pelvic pain. Etiological factors can vary. It is both gynecological disease and vascular lesions due to systemic manifestations, hormonal imbalance, inflammatory processes, genetic predisposition, anatomical and physiological features, etc. Pathogenetic varicose vein transformation follows two basic principles: valve insufficiency and development of obstructive disorders. This in turn leads to pathological reflux and stagnation in the pelvic venous system. The main reason for women's approach to specialists is chronic pelvic pain, which is characterized as non-cyclical pain lasting more than 6 months, localized in the small pelvis, reducing the quality of life of the patient. The main difficulty lies precisely in differential diagnosis. Under the mask of pelvic pain syndrome may hide a huge number of both gynecological and non-gynecological diseases. Timely assumption and adequate diagnostic routing of the patient will allow establishing the correct diagnosis faster and reducing the risk of diagnostic, and as a consequence, therapeutic errors. Conclusion. Despite the fact that pelvic varicose vein disease is a known disease and has been studied for many years, there is still no clear idea about the nature of the disease. This disease is multi-disciplinary in nature, and requires a coordinated and continuous work of specialists. Due to the lack of specificity in the clinical picture and reliable markers of the disease, many women experience a long and difficult journey from primary consultation to the final diagnosis.
\end{abstract}

Key words: pelvic varicose vein disease, chronic pelvic pain syndrome.

For reference: Minullina NK, Akhmetova DI. Modern understanding of pelvic varicose vein disease in the structure of chronic pelvic pain syndrome: diagnosis and treatment (review). The Bulletin of Contemporary Clinical Medicine. 2020; 13 (2): 62-69. DOI: 10.20969/VSKM.2020.13(2).62-69.

о данным Всемирной организации здравоохранения (ВО3), обращаемость женщин к специалистам с жалобой на тазовые боли составляет более $60-65 \%$ в год. На современном этапе развития гинекологии синдром хронической тазовой боли (ХТБ) имеет особую актуальность. Это связано с тем, что боль является клиническим проявлением огромного количества заболеваний, и только в 
редких случаях ее характер носит специфичность. Женщинам, которые страдают хронической болью в области малого таза, чаще устанавливается неверный диагноз, вследствие чего они получают некомпетентную терапию. Это не приносит облегчения пациентке и снижает качество ее жизни [1].

Ведущей причиной развития ХТБ является варикозное расширение вен малого таза. Результаты проведенных исследований на распространенность варикозной болезни вен таза (ВБВТ) говорят о том, что данная патология встречается у 5,4-25,6\% женщин репродуктивного возраста (в России - 15,4\%, в Великобритании - 24\%, в США - 20\%), которая увеличивается до $80 \%$ встречаемости в пременопаузу [2, 3].

Несмотря почти на вековую историю изучения ВБВТ, окончательного мнения по поводу этиологии, патогенеза, этапов диагностики и лечения так и не установлено.

Этиология. Предрасполагающими фракторами развития варикозного расширения вен могут служить как гинекологические, так и системные и сосудистые проявления, анатомические, физиологические и гормональные особенности женского организма. Четкого представления об этиологии на данный момент не сформировано. Однако в преобладающем большинстве пусковым механизмом служат многократные беременности и роды в анамнезе (у 97\% пациенток). Это объясняется сдавление беременной маткой магистральных сосудов (подвздошных и нижних полых вен). Также в период беременности увеличивается уровень прогестерона в крови, что ведет к снижению тонуса сосудистой стенки и избыточному растяжению вен. На последних сроках беременности объем циркулирующей крови увеличивается почти на $15 \%$. Стенка сосуда не способна выдержать избыточного давления и начинает ремоделироваться, что приводит к варикозной трансформации вен таза. Снижение тонуса сосудистой стенки могут провоцировать и другие гормональные перестройки: период полового созревания, менопауза, нарушение менструального цикла - гипоэстрагения, гормонозависимая терапия и контрацепция [4].

Некоторые случаи варикозного расширения вен малого таза были связаны с механическими причинами сжимающего характера и сдавлением. Это могут быть неправильное положение матки, вызывающее перегибание яичниковых вен, синдром Щелкунчика, когда левая почечная вена сжимается между аортой и верхней брыжеечной артерией, синдром Мея-Тернера и т.д. [5, 6].

К другим гинекологическим патологиям, которые вызывают ВБВТ, относят хронические воспалительные заболевания придатков матки, эндометриоз, спаечные процессы после оперативных вмешательств и перенесенных инфекционных процессов, опухоли матки и яичников [2].

Достоверно установлено, что в 50\% ВБВТ имеет генетическое обоснование. Выявлена связь между мутациями генов TIE2, NOTCH3, наличием гена FOXC2, уровнем тромбомодулина и развитием варикозной трансформации сосудов. Эти особен- ности ведут к структурным изменениям клапанного аппарата и стенки сосуда. Запускаются звенья патогенетических реакций, и развивается клиника варикозного расширения вен [7].

Патогенез. В настоящее время принято выделять первичную и вторичную форму развития ВБВТ. В патогенезе первичной варикозной трансформации вен лежит клапанная недостаточность венозных сосудов. Ее, в свою очередь, делят на врожденную и приобретенную [5]. Основным механизмом развития является рефлюкс крови и гипертензии в венозных сплетениях малого таза. При приобретенной клапанной недостаточности гемодинамические нарушения наблюдаются вследствие обструкции проксимального русла и гибели клапанов гонадных вен. К формированию обструкции относят следующие причины: кольцевая почечная вена, полное и неполное удвоение почечной вены, органический стеноз почечной вены, синдром Мея-Тернета, стеноз яичниковых вен, аортально-мезентеральный пинцет и др., которые проявляются чаще в период полового созревания и формируются аналогично врожденному или приобретенному рено-гонадному рефлексу. Первичная форма в виде рефлюкса и обтурации встречается в 65-70\% случаев [8].

Вторичная форма развития ВБВТ связана с наличием у пациентки гинекологической патологии (опухоли органов малого таза, эндометриоз и др.). Варикозная трансформация наблюдается на фроне интактных яичниковых вен. Встречаемость данной формы составляет 15-30\% [4] .

Нельзя забывать, что гонадные вены анатомически асимметричны. Яичниковые вены берут свое начало из сплетения в широкой связке около яичника и маточной трубы. Левая яичниковая вена дренируется в левую почечную вену под прямым углом, в то время как правая яичниковая вена дренируется непосредственно в нижнюю полую вену под косым углом. Это анатомическое различие отвечает за относительно слабую гемодинамику в левой яичниковой вене и считается причиной частого развития венозной патологии слева. Вариации гонадных вен могут отражать сложный эмбриогенез в этой области. Гонадная вена представляет численные вариации, а также вариации в ее месте дренажа, которые приписываются различным патологическим состояниям, таким как варикоцеле (у мужчин), синдром тазового застоя и т.д. [5, 7, 8].

Основными течениями ВБВТ являются варикозное расширение вен малого таза, промежности и вульвы и синдром тазового венозного полнокровия. Механизм варикозного расширения встречается у $30-35 \%$ женщин во время беременности. Этиопатогенетические аспекты сходны с развитием варикозного расширения вен нижних конечностей. Прогрессирование усугубляется компрессией беременной маткой магистральных вен забрюшинного пространства, особенно это наблюдается после второго триместра беременности [6]. На данный момент частота встречаемости синдрома тазового венозного полнокровия, согласно некоторым исследованиям, примерно равна 4\%. В основе этого синдрома лежит клапанная недостаточность яичниковых вен, при- 
водящая к сбросу крови и развитию повышенного давления в венозных сплетениях малого таза.

Клиническая картина ВБВТ неспецифична. Это является основной проблемой диагностики и дифференциальной диагностики данного заболевания. Ведущими проявлениями являются болевой синдром, варикозный синдром, нарушение функции тазовых органов и психоэмоциональный синдром. Первое, что заставляет женщин задуматься и обратится к гинекологу, является боли (пелвагии). Наиболее частой локализацией боли при ВБВТ является область таза с иррадиацией в пояснично-крестцовую и паховую область. Боль обычно описывается как более интенсивная на одной стороне, хотя при тщательном опросе, большинство пациенток также отмечают боль на контралатеральной стороне. Так же боли и дискомфорт могут быть в гипогастральной зоне с иррадиацией в область таза, промежностей и бедер. Частота такого рода болей варьируется от 60 до 80\%. Боль характеризуется как ноющая, тянущая, тупая, усиливается при длительном положении сидя или стоя, фризической нагрузке (53\%), которая постепенно купируется при отдыхе в горизонтальном положении с приподнятыми кверху ногами. ВБВТ носит гормонозависимый характер, поэтому болевой синдром может усиливаться во вторую фазу менструального цикла и при приеме гормональных препаратов [2].

Нарушение функции тазовых органов у больных ВБВТ может проявляться диспареунией (52\%), нарушением менструального цикла и выраженным предменструальным синдромом (19\%), дизурическими нарушениями (22\%), бесплодием и т.д. Боли во время полового акта (диспареуния) и после него встречаются при многих заболеваниях, однако следует обратить внимание, что при ВБВТ дискомфорт может сохранятся более нескольких суток, что серьезно подрывает качество жизни пациентки. Менструация чаще нерегулярная и длительная (5-7 дней) с большим количеством выделений. Дизурические нарушения характеризуются учащенным мочеиспусканием на фоне усиления болей. При наличии предрасположенности к развитию ВБВТ у женщины первые признаки могут наблюдаться еще в период полового созревания. Проявление выражается только лишь в обильных слизистых выделениях из половых путей. Именно на основании этого девушек лечат от кольпита, которого у них может и не быть [5].

Варикозный синдром при первичном осмотре визуализировать удается довольно редко. Изменения вен проявляются вульварным варикозом (на наружных половых органах, промежности), в области паха, над лобком, ягодицах [6]. Чаще всего визуализировать изменения удается в период беременности, особенно на поздних сроках. При выявлении варикозных вен на этих участках пациентку следует направить на ультразвуковое исследование вен таза [9].

ВБВТ сильно подрывает качество жизни женщины. На фоне заболевания у них развивается психоэмоциональный синдром. Постоянные и длительные боли, нарушения в сексуальной сфере ведут к нарушению психического здоровья пациентки, которые проявляются тревожностью, утомляемостью, раздражительностью, вплоть до депрессии. Диспареуния, в свою очередь, выражается вагинизмом и боязнью полового контакта, что служит развитию семейных конфлликтов и ухудшению общего психосоматического состояния пациентки.

Диагностика. Принцип и последовательность диагностики ВБВТ на данный момент не установлен. Основная сложность в выявлении данного заболевания лежит в отсутствии специфичных клинических проявлений и в сочетании с другими заболеваниями, которые могут давать схожую симптоматику. Под маской описанных выше симптомов могут скрываться заболевания не только гинекологического профиля, но и урологические, хирургические, неврологические. Иногда эта боль не имеет никакой очевидной причины, и ее диагностика и лечение могут иметь значительные трудности, не исключается, что в некоторых случаях потребуется и помощь психиатра.

От степени прогрессирования и данных инструментального анализа выбирается тактика лечения пациентки. Тактику обследования условно можно разделить на три компонента:

1) сбор анамнеза, общий и гинекологический осмотр;

2) скрининговые методы исследования и лабораторные анализы;

3) инвазивные методы исследования.

При первичном обращении женщины к гинекологу следует собрать подробный анамнез и сопоставить клиническую картину с ВБВТ. Для этого желательно провести анкетирование с оценкой боли и других симптомов по шкале ВАШ. Далее следует провести бимануальное влагалищно-абдоминальное исследование и осмотр с помощью зеркал. Специфичными признаками будет наличие тяжей и узелков вен, обнаруженных при пальпации по внутренним стенкам таза, увеличенная, мягкой консистенции матка, гипертрофия и цианоз шейки матки и влагалища [2].

Если в результате осмотра устанавливаются предварительные признаки ВБВТ, пациентка подлежит направлению на ультразвуковое исследование сосудов таза и лабораторные анализы.

Ведутся поиски современных и эффрективных методов диагностики варикозной болезни вен таза. Устанавливаются связи развития заболевания и определенных маркеров, которые являются факторами повреждения эндотелия сосудов. В качестве таких маркеров рассматриваются атерогенные стимулы (гомоцистеин и липопротеиды низкой плотности) и изменения иммунного статуса (угнетение Т-звена иммунитета, повышение уровня провоспалительных цитокинов) [1]. Высокая концентрация гомоцистеина ведет к повреждению интимы венозных сосудов, повреждая эндотелий. Тем самым фрибронектин и липопртеины, которые входят в состав сосудистой стенки, окисляются и подвергаются частичному протеолизу. Это приводит к снижению прочности венозной стенки, образованию бляшек и перерастяжению венозных сосудов. 
Большое внимание уделяется изучению воспалительного компонента в патогенезе развития варикозного расширения вен. У больных отмечается увеличение провоспалительных цитокинов, нарушение клеточного и гуморального ответа, что говорит о развитии эндотелиального воспаления [7]. При комплексной терапии венотропными, иммунотропными препаратами и препаратами, нормализующие метаболический обмен (липидный спектр), наблюдалось ускоренное повышение венозного сосудистого тонуса, нормализация гемодинамики и общего состояния пациенток в отличие от монотерапии флеботропными препаратами $[9,10]$.

К скрининговому методу диагностики ВБВТ можно отнести ультразвуковое сканирование вен таза. Визуализация имеет решающее значение для оценки варикозного расширения вен таза как для диффференциации от других состояний, так и потому, что варикозное расширение вен таза может быть вторичным по отношению к серьезным основным патологиям, таким как обструкция, портальная гипертензия и сосудистые мальформации.

Метод ультразвукового сканирования вен таза (ультразвуковое ангиосканирование, УЗАС) неинвазивный, абсолютно безопасный для пациентки и экономичный. Однако следует учесть, что УЗАС только констатирует наличие варикозной трасформации вен таза. Он не дает точной картины характера поражения и уровня поражения [11]. Для УЗАС применяют трансабдоминальный датчик (подвздошная, нижняя полая вена, почечные и почечный сегмент гонадной вены) и трансвагинальный датчик (вены матки, параметрия, яичниковый сегмент гонадных вен). Исследование проводится в В-режиме, в цветовом допплеровском кодировании, импульсивном режиме. При цветном допплеровском картировании (ЦДК) все вены должны полностью прокрашиваться. В импульсно-волновом режиме при использовании пробы Вальсальвы выявляем ретроградный кровоток, появляющийся в случае клапанной недостаточности сосуда [12]. Диагноз ВБВТ устанавливается при визуализации признаков расширения вен (диаметр более 5 мм) и наличии патологического рефлюкса по пробе Вальсальвы [3, 13,14$]$.

В настоящее время широко применяются магнитно-резонансная томография (МРТ) и компьютерная томография (КТ) в диагностике ВБВТ, однако данные виды исследования больше носят диффреренциальный диагностический характер с целью исключения других патологий области малого таза, которые могут давать схожую симптоматику и клиническую картину. При использовании мультиспиральной компьютерной томографии или эмиссионной компьютерной томографии при ВБВТ венозные сосуды визуализируется как расширенные, извилистые трубчатые структуры вблизи матки, яичников и т.д. [4, 14]. Этот метод имеет преимущества в установлении мезаортальной компрессии левой почечной вены. При помощи компьютерной томографии можно прогнозировать целесообразность консервативного лечения и в ранние сроки направить пациентку на оперативные вмешательства, если они необходимы. Магнитно-резонансная томография имеет те же плюсы, что и компьютерная томография. Этот метод обладает меньшей лучевой нагрузкой. Хотя безопасность процедур МРТ во время беременности окончательно не доказана, риск подвергнуть развивающийся плод радиологическому методу визуализации, использующему ионизирующее излучение, вероятно больше, чем теоретический риск MPT $[11,15]$.

В случае проведения хирургического лечения ВБВТ или при отсутствии ответа на проводимую консервативную терапию, женщину направляют на следующий этап обследования, в ходе которого проводится фрлебография и селективная оварикография [16, 17].

Флебографические контрастные исследования, применяемые при исследовании ВБВТ, представлены тазовой фрлебографией и селективной оварикографией. Селективная оварикография на сегодняшний день является самым информативным и ключевым методом диагностики, особенно в установке клапанной недостаточности [3]. Выполняется путем контрастирования гонадных вен после их селективной катетеризации. Технология метода заключается в пункции правой бедренной вены по Сельдингеру, затем путем проведения катетера достигают яичниковые вены (левую и правую). Селективная оварикография дает полную картину структурных изменений в сосудах и отмечает характер их возникновения. Она визуализирует анатомические патологии и фоновые компоненты возникновения ВБВТ. Данный метод исследования дает возможность более достоверно оценить особенность патологического рефлекса и гемодинамических нарушений, оценить градиентное давление между нижней полой веной и левой почечной, повышение которого говорит о значимом стенозе. Несмотря на все преимущества, селективная оварикография обладает рядом недостатков. В первую очередь, фрлебография является инвазивным вмешательством и имеет лучевую нагрузку, которая негативно сказывается на фолликулярном аппарате яичников [16]. В настоящее время данный метод применяют в лечебных целях. Во время селективной оварикографии можно выполнить эмболизацию вен спиралью или склерозирующими препаратами [14].

В случае невозможности установлении верного диагноза и его дифференциации после всех приведенных выше методов исследования, прибегают к лапароскопии. Уровень точности и чувствительности данного метода исследования возрастает по мере выраженности патологического процесса в венозных сосудах, поэтому ряд специалистов рассматривают этот вариант как обязательный в плане диагностики ВБВТ. Основными недостатками данного метода исследования являются высокая степень инвазивности и вероятность развития осложнений, а также дороговизна [4].

Нельзя забывать, что хронические тазовые боли являются симптомом не только гинекологического профиля. Если в результате полного и качественного обследования специалистом у больной не было установлено какой-либо патологии, то пациентка 
должна быть направлена врачу другого профиля (хирург, уролог, невролог, проктолог).

Лечение. На данном этапе не сформировано четкого представления о лечебной тактике пациенток с варикозной болезнью вен малого таза. Выделяют два основных подхода: консервативная терапия и хирургические вмешательства. В зависимости от степени варикозного процесса, выраженности болевого синдрома, анатомических и физиологических особенностей, приводящих к развитию заболевания у конкретной женщины, прибегают к тому или иному методу лечения.

При первой степени варикоза без ярко выраженного болевого синдрома стартовая терапия носит консервативный характер. Она включает в себя изменения образа жизни, пищевого режима, компрессионной, а также медикаментозной терапии и фризиотерапевтических процедур [11]. Пациентке следует рекомендовать отказаться от подъема тяжестей, соблюдение режима труд-отдых, исключить длительное пребывание в вертикальном положении. В положении лежа следует выполнять ряд элементарных фризических упражнений («велосипед», «березка» и т.д.) в целях разгрузки и снижения гипертензии в венах малого таза и нижних конечностей. Компрессионная терапия снижает стаз крови, ускоряя венозный отток, носит профилактический эффрект (снижает риск тромбообразования), поэтому женщинам необходимо носить эластичный трикотаж 2-го компрессионного класса с воздействием на нижние конечности и переднюю брюшную стенку [2].

В арсенале гинекологов существует ряд препаратов, которые воздействуют на основные звенья патогенеза, тем самым, действуя системно, помогают пациентке улучшить качество жизни. Фармакотерапия должна носить комплексный характер и включать в себя венопротекторы (деосминсодержащие препараты и их комбинации), нестероидные противовоспалительные средства, антиоксиданты [10]. При соблюдении правильного режима приема медикаментов купируется или значительно снижается болевой синдром, уменьшаются явления тазового застоя. Вкупе с вышеуказанными рекомендациями применяются фризиопроцедуры. Выраженный положительный результат наблюдается при использовании электрофрореза с колализином [2].

В случае неэфффективности консервативной терапии, при 2-3-й стадиях варикозного процесса, выраженной клинической картине ВБВТ, наличии установленного рефлюкса, варикозе наружных половых органов проводится хирургическое лечение. Основные направления оперативных вмешательств можно разделить на резекцию вен и эндоваскулярные манипуляции (эмболизация спиралью, склерозирование) [13].

Резекция вен осуществляется двумя основным способами: внебрюшная резекция и черезбрюшная с использованием лапароскопического инструментария. В первом случае применяется доступ Пирогова. Сам сосуд выделяют на протяжении 10-15 см с последующей резекцией и перевязкой, а все притоки и сагиттальные вены подвергаются лигированию. Несмотря на высокую эфффективность и почти минимальное число рецидивов, данная методика достаточна травматична и носит серьезный косметический дефект, что следует учитывать, если оперируемая пациентка молодого возраста. При эндоскопическом лечении производится наложение пневмоперитонеума. После введения инструментов пациентку переводят в положение Транделенбурга и осуществляют резекцию вен длинной 8-10 см после предварительного клипирования. Преимущество лапароскопической операции: сокращение послеоперационного периода, быстрая реабилитация и удовлетворительный эстетический эффрект. Купирование тазовых болей или снижение их выраженности наблюдается в 95-100\% случаев в период от 3 нед до 4 мес после операции. Рецидивы наблюдаются, по данным различных источников, от 0 до 5\% [17].

Эндовазальные технологии прочно вошли в арсенал диагностических и лечебных мероприятий. В отношении ВБВТ на сегодняшний день применимы эмболизация и склеротерапия вен. Эндоваскулярная эмболизация осуществляется с применением спирали Джианкурко. Чаще всего данное вмешательство проводят уже на этапе выполнения селективной оварикографии или тазовой флебографии. Окклюзионную спираль устанавливают на уровне средней трети вены, тем самым обеспечивая прекращение ретроградного кровотока [14]. Эффрективность данного метода колеблется от 70 до 100\%, а риск развития рецидивов составляет 10-38\%, основной причиной которых является рефлюкс по коллатералям [16]. В литературе описано ряд осложнений после данной процедуры, в частности смещение спирали и ее миграция. При методе катетерной склеротерапии вен используют склерозанты жидких и пенных форм, а также сосудосуживающие вещества. Препараты вводят в дистальные отделы гонадных вен. Основное преимущество заключается в том, что склерозирующие вещества распространяются по притокам и коллатералям, тем самым снижая риск рецидива и повышая эффективность лечения. Несмотря на хорошие результаты проводимой терапии и оперативного лечения, примерно через 2 года у 21,7\% женщин после фрлебосклерозирования и у 12,5\% после комплексной терапии (операция+лечебно-профилактические мероприятия) наблюдается возобновление симптоматики ВБВТ, особенно синдрома тазовой боли. Положительные особенности эндоваскулярного лечения заключаются в малоинвазивности, быстрой скорости выполнения процедуры, быстрой реабилитации и высокой эстетичности [17].

Перспективным направлением лечения с применением эндоваскулярных технологий является методика «сэндвич». Особенность заключается в применении склерозантов и спирали вместе, локализуя спираль в средней трети гонадных вен. Точной информации об эффеективности пока нет, но ряд экспертов выделяют этот метод как стандарт лечения ВБВТ. Благодаря комбинированному использованию склерозирующих веществ и эмобилизацонной спирали достигается стойкое купирование болевого синдрома в короткий срок с минимальной вероятностью возобновления симптомов в будущем $[14,18]$. 
Одним из основных недостатков оперативного лечения является частота развития рецидивов. Флебологи разделяют их на «истинные» и «ложные». В первую группу относятся рецидивы, возникшие в результате погрешности в выполнении манипуляций. К «ложным» относится прогрессирование варикозной трансформации. Нельзя забывать, что хронические боли таза могут вызывать и другие заболевания, которые никак не связаны с варикозом. О рецидиве целесообразно говорить только в том случае, если патологический рефлюкс, тазовое венозное полнокровие и расширение вен установлены объективно в результате диагностических обследований. После проведенного оперативного лечения следует проводить профилактические мероприятия. Это объясняется тем, что ВБВТ носит системный характер, хирургия не может воздействовать на этиологические и патогенетические звенья развития варикозных изменений сосудов в целом. Из этого следует, что изменения строения венозной стенки и клапанного аппарата могут локализоваться как в ранее пораженных области, так и в абсолютного других участках. Чаще всего варикозная трансформация наблюдается на противоположной от оперированной стороне. Исходя из этого некоторые специалисты рекомендуют осуществлять хирургическое вмешательство двустороннего характера, несмотря на наличие патологических изменений сосудов [18].

Выводы. Несмотря на то что вопрос ВБВТ изучается уже более 100 лет, четко сформулированных представлений об основных этиопатогенетических механизмах, методах диагностики и лечения не имеется. Имея мощную техническую возможность как в диагностике, так и в лечении, многие женщины не получают должную помощь в связи с тем, что клиническая картина неспецифична, и поэтому период от первичного обращения до адекватного лечения может растянуться до нескольких лет.

Прозрачность исследования. Исследование не имело спонсорской поддержки. Авторы несут полную ответственность за предоставление окончательной версии рукописи в печать.

Декларация о финансовых и других взаимоотношениях. Все авторы принимали участие в разработке концепции, дизайна исследования и в написании рукописи. Окончательная версия рукописи была одобрена всеми авторами. Авторы не получали гонорар за исследование.

\section{ЛИТЕРАТУРА}

1. Клинико-лабораторные изменения при варикозной болезни малого таза у женщин / А.А. Семендяев, М.Б. Хамошина, П.М. Самчук [и др.] // Бюллетень ВСНЦ CO PAMH. - 2010. - № 6 (76). - C.60-62.

2. Шоста, А.В. Варикозная болезнь и хроническая венозная недостаточность в практике гинеколога / А.В. Шоста // Медицинские новости. - 2012. - № 10. - С.29-31.

3. Is pelvic vein incompetence associated with symptoms of chronic pelvic pain in women? / V. Hansrani, J. Morris, A. Caress [et al.] // Eur. J. Obstet. Gynecol Reprod. Biol. 2016. - Vol. 196. - P.21-25.

4. Durham, J.D. Pelvic congestion syndrome / J.D. Durham, L. Machan // Semin Intervent Radiol. - 2013. - Vol. 30 (4). P.372-380.
5. Pelvic congestion syndrome: etiology of pain, diagnosis, and clinical management / D. Phillips, A.R. Deipolyi, R.L. Hesketh [et al.] // J. Vasc. Interv. Radiol. - 2014. Vol. 25 (5). - P.725-733.

6. Алгоритм обследования женщин с синдромом тазовой боли при подозрении на варикозную болезнь вен таза / А.И. Гус, М.Б. Хамошина, С.М. Бачурина [и др.] // Сибирский медицинский журнал. - 2011. - № 6. - С.83-85.

7. Pathogenesis of varicose veins / R. Oklu, R. Habito, M. Mayr [et al.] // J. Vasc. Interv. Radiol. - 2012. Vol. 23 (1). - P.33-39.

8. Ahmed, O. Endovascular stent placement for May-Thurner syndrome in the absence of acute deep vein thrombosis / O. Ahmed, J. Ng, M. Patel // J. Vasc. Interv. Radiol. 2016. - Vol. 27 (2). - P.167-173.

9. Chronic hip pain as a presenting symptom in pelvic congestion syndrome / C Huang, J. Shelkey, H. Singh [et al.] // J. Vasc. Interv. Radiol. - 2013. - Vol. 24 (5). P.753-755.

10. Эффрективность иммунокорригирующей терапии при варикозной болезни вен малого таза у женщин/ А.И. Гус, М.Б. Хамошина, С.М. Бачурина [и др.] // Бюллетень ВСНЦСО РАМН. - 2013. - № 6 (94). - С.24-28.

11. Pelvic venous insufficiency: imaging diagnosis, treatment approaches, and therapeutic issues / M.G. Knuttinen, K. Xie, A. Jani [et al.] // AJR Am. J. Roentgenol. - 2015. Vol. 204 (2). - P.448-458.

12. Фомина, Е.Е. Методология ультразвукового исследования при варикозной болезни вен таза / Е.Е. Фомина, Р.В. Ахметзянов, М.Г. Тухбатуллин // Практическая медицина. - 2016. - № 9. - С.53-58.

13. Российские клинические рекомендации по диагностике и лечению хронических заболеваний вен / Ю.М. Стойко, А.И. Кириенко, И.И. Затевахин [и др.] // Флебология. - 2018. - № 3 - С.148-240.

14. The relationship between pelvic vein incompetence and chronic pelvic pain in women: systematic reviews of diagnosis and treatment effectiveness / R. Champaneria, L. Shah, J. Moss [et al.] // Health Technol. Assess. 2016. - Vol. 20 (5). - P.1-18.

15. Role of trans vaginal ultrasound and Doppler in diagnosis of pelvic congestion syndrome / K. Sharma, M.K. Bora, J. Varghese [et al.] // J. Clin. Diagn. Res. - 2014. Vol. 8 (7). - P.5-12.

16. Pelvic congestion syndrome-associated pelvic pain: a systematic review of diagnosis and management / D. Hahn, J.F. Steege // Obstet. Gynecol. Surv. - 2010. Vol. 65 (5). - P.332-340.

17. Принципы хирургического лечения варикозной болезни вен таза / С.Г. Гаврилов, А.И. Кириенко, В.И. Ревякин [и др.] // Вестник РГМУ. - 2009. - № 5. - С.11-18.

18. Borghi, C. Pelvic congestion syndrome: the current state of the literature / C. Borghi, L. Dell'Atti // Arch. Gynecol. Obstet. - 2016. - Vol. 293 (2). - P.291-301.

\section{REFERENCES}

1. Semendyaev AA, Hamoshina MB, Samchuk PM. Klinikolaboratornie izmeneniya pri varikoznoi bolezni malogo taza $u$ jenschin [Clinical and laboratory changes in women with pelvic varicose disease]. Byulleten VSNC SO RAMN [Bulletin of SB RAMS]. 2010; 6 (76): 60-62.

2. Shosta AV. Varikoznaya bolezn i hronicheskaya venoznaya nedostatochnost $v$ praktike ginekologa [Varicose disease and chronic venous insufficiency in the gynecology practice]. Medicinskienovosti [Medicalnews]. 2012; 10: 29-31.

3. Hansrani V, Morris J, Caress A. Is pelvic vein incompetence associated with symptoms of chronic pelvic pain in women? Eur J Obstet Gynecol Reprod Biol. 2016; 196: $21-25$. 
4. Durham JD, Machan L. Pelvic congestion syndrome. Semin Intervent Radiol. 2013; 30 (4): 372-380.

5. Phillips D, Deipolyi AR, Hesketh RL. Pelvic congestion syndrome: etiology of pain, diagnosis, and clinical management. J Vasc Interv Radiol. 2014; 25 (5): 725-733.

6. Gus AI, Hamoshina MB, Bachurina SM. Algoritm obsledovaniya jenschin s sindromom tazovoi boli pri podozrenii na varikoznuyu bolezn ven taza [The algorithm of examination of women the syndrome of pelvic pain in suspicion for varicose veins of small pelvis]. Sibirskii medicinskii jurnal [The Siberian Scientific Medical Journal]. 2011; 6: 83-85.

7. Oklu R, Habito R, Mayr M, Watkins MT, et al. Pathogenesis of varicose veins. J Vasc Interv Radiol. 2012; 23 (1): 33-39.

8. Ahmed O, Ng J, Patel M. Endovascular stent placement for May-Thurner syndrome in the absence of acute deep vein thrombosis. J Vasc Interv Radiol. 2016; 27 (2): 167-173.

9. Huang C, Shelkey J, Singh H. Chronic hip pain as a presenting symptom in pelvic congestion syndrome. J Vasc Interv Radiol. 2013; 24 (5): 753-755.

10. Gus AI, Khamoshina MB, Bachurina SM. Effektivnost immunokorregiruyuschei terapii pri varikoznoi bolezni ven malogo taza u jenschin [Efficacy of immune correcting therapy at varicose vein disease of pelvis minor in women]. Byulleten VSNCSO RAMN [Bulletin of SB RAMS]. 2013; 6 (94): 24-28.

11. Knuttinen MG, Xie K, Jani A. Pelvic venous insufficiency: imaging diagnosis, treatment approaches, and therapeutic issues. AJR Am J Roentgenol. 2015; 204 (2): 448-458.
12. Fomina EE, Ahmetzyanov RV, Tuhbatullin MG. Metodologiya ultrazvukovogo issledovaniya pri varikoznoi bolezni ven taza [Methodology of ultrasound investigation of pelvic varicose disease]. Prakticheskaya medicina [Practical medicine]. 2016; 9: 53-58.

13. Ctoyko YuM, Kiriyenko AI, Zatevakhin II. Rossiyskie klinicheskie rekomendatsii po diagnostike i lecheniyu khronicheskikh zabolevaniy ven [Russian clinical guidelines for diagnosis and treatment of chronic venous diseases]. Phlebology [Flebologiya]. 2018; 3: 148-240.

14. Champaneria R, Shah L, Moss J, Gupta JK, Birch J, Middleton LJ, Daniels JP. The relationship between pelvic vein incompetence and chronic pelvic pain in women: systematic reviews of diagnosis and treatment effectiveness. Health Technol Assess. 2016; 20 (5): 1-18.

15. Sharma K, Bora MK, Varghese J, Malik G, Kuruvilla R. Role of trans vaginal ultrasound and Doppler in diagnosis of pelvic congestion syndrome. J Clin Diagn Res. 2014; 8 (7): 5-12.

16. Hahn D, Steege JF. Pelvic congestion syndromeassociated pelvic pain: a systematic review of diagnosis and management. Obstet Gynecol Surv. 2010; 65 (5): 332-340.

17. Gavrilov SG, Kirienko AI, Revyakin VI. Principi hirurgicheskogo lecheniya varikoznoi bolezni ven taza [Principles of surgical treatment of pelvic veins' varicose disease]. VestnikRGMU [Bulletinof RGMU]. 2009; 5: 11-15.

18. Borghi C, Dell'Atti L. Pelvic congestion syndrome: the current state of the literature. Arch Gynecol Obstet. 2016; 293 (2): 291-301. 